\title{
A two-variable approach to solve the polynomial Lyapunov equation
}

\author{
Ralf Peeters, Paolo Rapisarda* \\ Department of Mathematics, Universiteit Maastricht, P.O. Box 616, 6200 MD Maastricht, Netherlands
}

Received 1 January 2000; received in revised form 1 August 2000

\begin{abstract}
A two-variable polynomial approach to solve the one-variable polynomial Lyapunov equation is proposed. Lifting the problem from the one-variable to the two-variable context allows to use Faddeev-type recursions in order to solve the polynomial Lyapunov equation in an iterative fashion. The method is especially suitable for applications requiring exact or symbolic computation. (c) 2001 Elsevier Science B.V. All rights reserved.
\end{abstract}

Keywords: Two-variable polynomial matrices; Polynomial Lyapunov equation; Faddeev's method; Quadratic differential forms; Symbolic computation

\section{Introduction}

Let $R$ be a real $q \times q$ polynomial matrix in the indeterminate $\xi$, i.e.,

$R(\xi)=R_{0}+R_{1} \xi+\cdots+R_{L} \xi^{L}$,

where $R_{i}$ is a real $q \times q$ constant matrix for $i=1, \ldots, L$, such that the polynomial $\operatorname{det}(R)$ is not identically zero. Let $Z$ be a $q \times q$ polynomial matrix in the indeterminate $\xi$ satisfying $Z(\xi)=Z(-\xi)^{\mathrm{T}}$. The equation

$R(-\xi)^{\mathrm{T}} X(\xi)+X(-\xi)^{\mathrm{T}} R(\xi)=Z(\xi)$

in the unknown $q \times q$ polynomial matrix $X$ is called the polynomial Lyapunov equation (PLE).

The PLE arises in various areas of mathematical systems theory. For example, it plays a role in the computation of integrals of quadratic functionals of the variables of a system and their derivatives (see

\footnotetext{
* Corresponding author.

E-mail addresses: ralf.peeters@math.unimaas.nl (R. Peeters), p.rapisarda@math.unimaas.nl (P. Rapisarda).
}

$[1,11,12])$. The PLE also arises in stability theory for higher-order differential equations as a generalization of the usual Lyapunov stability test for first-order systems (see $[15,8])$.

In this paper we concentrate on the particular case in which $Z$ attains the form $Z(\xi)=Q(-\xi)^{\mathrm{T}} \Sigma Q(\xi)$, where for some positive integer $p, Q$ is a real $p \times q$ polynomial matrix in the indeterminate $\xi$ such that $Q R^{-1}$ is a matrix of strictly proper rational functions and $\Sigma$ is a $p \times p$ signature matrix, i.e., a diagonal matrix with \pm 1 entries on the main diagonal. Such form of the PLE is commonly encountered in practice. Thus, we will at first be concerned with solving the equation

$$
R(-\xi)^{\mathrm{T}} X(\xi)+X(-\xi)^{\mathrm{T}} R(\xi)=Q(-\xi)^{\mathrm{T}} \Sigma Q(\xi)
$$

and in Section 4, we will show that this enables us to also solve the general form of the equation.

In this paper a new algorithm to solve the PLE is proposed which differs in several respects from previous algorithms described in the literature. First of all, the proposed approach involves lifting the problem 
from the one-variable polynomial context in which it is originally formulated to a two-variable context. (For another successful application of this lifting technique, see [13].) This brings along the two-fold advantage of exploiting the structure of the PLE (1.1) and of shifting attention from the PLE to an associated equation, the so-called lifted polynomial Lyapunov equation (LPLE), which is defined on a finite-dimensional vector space instead.

Second, the actual algorithm for solving the PLE proceeds by solving the LPLE by an iterative method which is inspired by the Faddeev sequence-based approach of [6] to solve matrix Sylvester and Lyapunov equations. Thus designed, the method turns out to be especially suited for applications requiring symbolic or exact computation [9]. In contrast to the available algorithms described in the literature, the given method proceeds directly from the polynomial matrices $R$ and $Q$ defining the PLE and requires no further preprocessing or transformation to some canonical form. An iterative approach for the solution of the PLE in the scalar case has previously been used in $[5,8]$ in the context of stability tests for scalar polynomials. However, the algorithm presented in this paper can instead be applied to both scalar and multivariable problems. This generality stems from the possibility of using the calculus developed in [14] for dealing with quadratic differential forms, i.e., with quadratic functionals of the variables of a system and their derivatives, which are intimately related to two-variable polynomial matrices.

A few words on notation. In this paper, as in [7], a polynomial matrix $R(\xi)$ in the indeterminate $\xi$ is called nonsingular if the polynomial $\operatorname{det}(R(\xi)) \neq 0$. For such $R$ the inverse is denoted with $R(\xi)^{-1}$, the transpose with $R(\xi)^{\mathrm{T}}$ and the transpose of the inverse with $R(\xi)^{-\mathrm{T}}$. Following [14], if a polynomial matrix $Z(\xi)$ satisfies $Z(\xi)=Z(-\xi)^{\mathrm{T}}$ it is called para-Hermitian. If $Z(\xi)$ satisfies $Z(\xi)=-Z(-\xi)^{\mathrm{T}}$ it is called skew-para-Hermitian.

The paper is organized as follows. In Section 2 we introduce several concepts regarding polynomial matrices and shifts in a single variable. In Section 3 these notions are extended to the case of symmetric two-variable polynomials and we define the Lyapunov operator as a two-variable shift operator on a particular finite-dimensional vector space. In Section 4 we complete the development of our framework for the study of the PLE. Here, the PLE is lifted to the two-variable context, thus giving rise to the LPLE. In Section 5 the Lyapunov operator is used to formulate a recursive algorithm to compute a solution to the LPLE which is inspired by Faddeev's method. From such solution a so-called $R$-canonical solution to the PLE is easily obtained. In Section 6 the algorithm is illustrated by a worked example. A section containing final remarks concludes the paper. All the proofs are given in the appendix.

\section{2. $R$-equivalence and the one-variable shift operator}

In this section we introduce the notions concerning polynomial matrices in a single variable needed in the rest of this paper. The concepts introduced in this section are not new, although the terminology may differ from that used elsewhere. See [2,3,14] for a collection of similar results.

Let $R$ be an element of $\mathbb{R}^{q \times q}[\xi]$, the set of $q \times q$ polynomial matrices in the indeterminate $\xi$. Assume that $R$ is nonsingular, i.e., the polynomial $\operatorname{det}(R)$ does not vanish identically. Then it is easily established (cf., e.g., [2, Chapter 2]) that $R$ induces an equivalence relation on the set $\mathbb{R}^{1 \times q}[\xi]$ as follows.

Definition 2.1. Two $1 \times q$ polynomial matrices $D_{1}, D_{2} \in \mathbb{R}^{1 \times q}[\xi]$ are called $R$-equivalent if there exists a polynomial matrix $P \in \mathbb{R}^{1 \times q}[\xi]$ such that $D_{1}-D_{2}=P R$. A polynomial matrix $D \in \mathbb{R}^{1 \times q}[\xi]$ is called $R$-canonical if $D R^{-1}$ is a strictly proper rational matrix.

It is easily shown that every $1 \times q$ polynomial matrix $D$ admits a unique $R$-canonical polynomial matrix $D^{\prime}$ which is $R$-equivalent to $D$. Such matrix $D^{\prime}$ can be computed as $D^{\prime}=S R=D-P^{\prime} R$, where $P^{\prime}$ denotes the polynomial part and $S$ the strictly proper rational part of $D R^{-1}=P^{\prime}+S$. We denote the $R$-canonical representative $D^{\prime}$ of the $R$-equivalence class of $D$ with $D \bmod R$. Uniqueness follows from the observation that if $D^{\prime \prime}=D-P^{\prime \prime} R$ is another $R$-canonical matrix $R$-equivalent to $D$ with $P^{\prime \prime}$ polynomial, then $\left(D^{\prime}-\right.$ $\left.D^{\prime \prime}\right) R^{-1}=P^{\prime}-P^{\prime \prime}$ is both strictly proper and polynomial, hence zero.

The subset of $\mathbb{R}^{1 \times q}[\xi]$ consisting of all $R$-canonical polynomial matrices is denoted by $\mathscr{C}_{R}^{1 \times q}[\xi]$. It is easily verified that $\mathscr{C}_{R}^{1 \times q}[\xi]$ is a vector space that can be identified naturally with the vector space of $R$-equivalence classes in $\mathbb{R}^{1 \times q}[\xi]$.

Proposition 2.2. The space $\mathscr{C}_{R}^{1 \times q}[\xi]$ is a finite-dimensional vector space over $\mathbb{R}$ of dimension $n=$ $\operatorname{deg}(\operatorname{det}(R))$. 
We proceed to define the polynomial shift operator $S$ acting on $\mathscr{C}_{R}^{1 \times q}[\xi]$ (see also [2]).

Definition 2.3. The one-variable polynomial shift operator $S: \mathscr{C}_{R}^{1 \times q}[\xi] \rightarrow \mathscr{C}_{R}^{1 \times q}[\xi]$ is defined as $S(D(\xi)):=\xi D(\xi) \bmod R(\xi)$.

Observe that $S$ is linear. Its characteristic polynomial is entirely determined by $\operatorname{det}(R)$, as described in the following proposition which generalizes $[2$, Theorem 4-6].

Proposition 2.4. The characteristic polynomial $\chi_{S}(\xi)$ of $S$ is equal to $\chi_{S}(\xi)=\operatorname{det}(R(\xi)) / r_{0}$, where $r_{0}$ denotes the leading coefficient of $\operatorname{det}(R(\xi))$.

The definition of the shift $S$ can be extended from $\mathbb{R}^{1 \times q}[\xi]$ to $\mathbb{R}^{k \times q}[\xi]$ in a row-by-row manner. The concepts of $R$-equivalence and $R$-canonicity are extended likewise. The subspace of $R$-canonical elements of $\mathbb{R}^{k \times q}[\xi]$ is denoted by $\mathscr{C}_{R}^{k \times q}[\xi]$.

\section{3. $R$-equivalence and the two-variable shift operator}

In this section we study $R$-equivalence, $R$-canonicity and the shift operator on spaces of symmetric polynomial matrices in two variables. The material of this section is in part a review of notions developed in the context of quadratic differential forms, see [14]. The section contains also new material, most notably the study of the Lyapunov operator $\mathscr{L}_{R}$.

We denote with $\mathbb{R}^{q \times q}[\zeta, \eta]$ the set consisting of $q \times q$ real polynomial matrices in the two indeterminates $\zeta$ and $\eta$. Observe that $\mathbb{R}^{q \times q}[\zeta, \eta]$ is a vector space over $\mathbb{R}$. A polynomial matrix $\Phi \in \mathbb{R}^{q \times q}[\zeta, \eta]$ is called symmetric if $\Phi(\zeta, \eta)=\Phi(\eta, \zeta)^{\mathrm{T}}$. The subspace of $\mathbb{R}^{q \times q}[\zeta, \eta]$ consisting of its symmetric elements is denoted with $\mathbb{R}_{\mathrm{sym}}^{q \times q}[\zeta, \eta]$.

Let $R \in \mathbb{R}^{q \times q}[\xi]$ be nonsingular. It is straightforward to see that $R$ induces an equivalence relation on $\mathbb{R}_{\mathrm{sym}}^{q \times q}[\zeta, \eta]$ in the following way.

Definition 3.1. Two symmetric $q \times q$ polynomial matrices $\Phi_{1}, \Phi_{2} \in \mathbb{R}_{\mathrm{sym}}^{q \times q}[\zeta, \eta]$ are called $R$-equivalent if there exists a polynomial matrix $P \in \mathbb{R}^{q \times q}[\zeta, \eta]$ such that $\Phi_{1}(\zeta, \eta)-\Phi_{2}(\zeta, \eta)=R(\zeta)^{\mathrm{T}} P(\zeta, \eta)+P(\eta, \zeta)^{\mathrm{T}} R(\eta)$. A polynomial matrix $\Phi \in \mathbb{R}_{\mathrm{sym}}^{q \times q}[\zeta, \eta]$ is called $R$ canonical if $R(\zeta)^{-\mathrm{T}} \Phi(\zeta, \eta) R(\eta)^{-1}$ is a strictly proper rational matrix.
It is not difficult to show that every $\Phi \in \mathbb{R}_{\mathrm{sym}}^{q \times q}[\zeta, \eta]$ admits a unique $R$-canonical two-variable symmetric polynomial matrix $\Phi^{\prime}$ which is $R$-equivalent to $\Phi$. Such matrix $\Phi^{\prime}$ can be obtained as follows. Compute a factorization of $\Phi$ as $\Phi(\zeta, \eta)=M(\zeta)^{\mathrm{T}} N(\eta)$ (see $[14,10]$ or the proof of Proposition 3.2 below for how to perform such factorization). Now define $\Phi^{\prime}(\zeta, \eta):=M^{\prime}(\zeta)^{\mathrm{T}} N^{\prime}(\eta)$, where $M^{\prime}=M \bmod R$ and $N^{\prime}=N \bmod R$ (in the sense of one-variable $R$-equivalence). We denote the $R$-canonical representative $\Phi^{\prime}$ of the $R$-equivalence class of $\Phi \in \mathbb{R}_{\mathrm{sym}}^{q \times q}[\zeta, \eta]$ with $\Phi \bmod R$. The subset of $\mathbb{R}_{\mathrm{sym}}^{q \times q}[\zeta, \eta]$ consisting of all $R$-canonical two-variable symmetric polynomial matrices is denoted by $\mathscr{C}_{R, \mathrm{sym}}^{q \times q}[\zeta, \eta]$. It is easily seen that $\mathscr{C}_{R \text {,sym }}^{q \times q}[\zeta, \eta]$ is a vector space that can be identified naturally with the vector space of $R$-equivalence classes in $\mathbb{R}^{q \times q}[\zeta, \eta]$. The dimension of $\mathscr{C}_{R}^{q \times q}[\zeta, \eta]$ is established as follows.

Proposition 3.2. The space $\mathscr{C}_{R, \mathrm{sym}}^{q \times q}[\zeta, \eta]$ is a finite-dimensional vector space over $\mathbb{R}$ of dimension $n(n+$ $1) / 2$, where $n=\operatorname{deg}(\operatorname{det}(R))$.

We proceed to define the two-variable shift operator $\mathscr{L}_{R}$ acting on the space $\mathscr{C}_{R, \text { sym }}^{q \times q}[\zeta, \eta]$ of $R$-canonical two-variable symmetric polynomial matrices. For reasons that will become evident in the next section, we shall refer to this operator $\mathscr{L}_{R}$ as the Lyapunov operator associated with $R$.

Definition 3.3. The Lyapunov operator $\mathscr{L}_{R}$ : $\mathscr{C}_{R, \text { sym }}^{q \times q}[\zeta, \eta] \rightarrow \mathscr{C}_{R \text {, sym }}^{q \times q}[\zeta, \eta]$ is defined as

$\mathscr{L}_{R}(\Phi(\zeta, \eta)):=(\zeta+\eta) \Phi(\zeta, \eta) \bmod R$.

Observe that $\mathscr{L}_{R}$ is linear. Its characteristic polynomial is entirely determined by $\operatorname{det}(R)$ as described in the following proposition.

Proposition 3.4. The characteristic polynomial $\chi_{\mathscr{L}_{R}}$ of the Lyapunov operator $\mathscr{L}_{R}$ is given by

$\chi \mathscr{L}_{R}(\xi):=\prod_{1 \leqslant i \leqslant j \leqslant n}\left(\xi-\left(\lambda_{i}+\lambda_{j}\right)\right)$,

where $n=\operatorname{deg}(\operatorname{det}(R))$ and $\lambda_{1}, \ldots, \lambda_{n}$ are the zeros of the polynomial $\operatorname{det}(R)$.

\section{The lifted polynomial Lyapunov equation}

In this section we complete the set up of our framework for the solution of the PLE. We begin by 
lifting the problem of computing a solution to the PLE from the one-variable polynomial context in which it is originally formulated, to a two-variable polynomial context. To this end, we now introduce a two-variable polynomial equation associated with the matrices $R$, $Q$ and $\Sigma$ which determine the PLE. The equation

$(\zeta+\eta) Y(\zeta, \eta) \bmod R=Q(\zeta)^{\mathrm{T}} \Sigma Q(\eta)$

in the unknown $R$-canonical symmetric two-variable polynomial matrix $Y \in \mathscr{C}_{R, \text { sym }}^{q \times q}[\zeta, \eta]$ is called the lifted polynomial Lyapunov equation (LPLE). Solvability of the PLE is equivalent to solvability of the LPLE, as the following proposition shows.

Proposition 4.1. Let $R \in \mathbb{R}^{q \times q}[\xi]$ be nonsingular, let $Q \in \mathbb{R}^{p \times q}[\xi]$ be $R$-canonical and let $\Sigma$ be a $p \times p$ signature matrix. Then the following two statements are equivalent:

1. There exists a solution $X \in \mathbb{R}^{q \times q}[\xi]$ to the PLE (1.1).

2. There exists a solution $Y \in \mathscr{C}_{R, \mathrm{sym}}^{q \times q}[\zeta, \eta]$ to the LPLE (4.1).

The next proposition characterizes the space of solutions to the PLE and generalizes [14, Theorem 4.8].

Proposition 4.2. Let $R \in \mathbb{R}^{q \times q}[\xi]$ be nonsingular, let $Q \in \mathbb{R}^{p \times q}[\xi]$ be $R$-canonical and let $\Sigma$ be a $p \times p$ signature matrix. Let $\mathscr{X}_{R} \subset \mathscr{C}_{R}^{q \times q}[\xi]$ be the set of all $R$-canonical solutions to the PLE and let $\mathscr{S}$ be the set of all skew-para-Hermitian polynomial matrices $S \in \mathbb{R}^{q \times q}[\xi]$, i.e., matrices for which $S(\xi)=-S(-\xi)^{\mathrm{T}}$. Then the space of all solutions to the PLE is given by

$\mathscr{X}_{R} \oplus \mathscr{S} R=\left\{X+S R \mid X \in \mathscr{X}_{R}\right.$ and $\left.S \in \mathscr{S}\right\}$.

Observe that Proposition 4.2 implies that the PLE has a solution if and only if it has an $R$-canonical solution. Consequently, the search for a solution to the $P L E$ can be restricted from the infinite-dimensional space $\mathbb{R}^{q \times q}[\xi]$ to the finite-dimensional space $\mathscr{C}_{R}^{q \times q}[\xi]$ of R-canonical polynomial matrices.

From the proof of Proposition 4.1, a method for constructing a solution $X$ to the PLE from a given solution $Y$ to the LPLE becomes apparent. Indeed, if $Y$ is a solution to the LPLE then by definition of $R$-equivalence there exists a polynomial matrix $P \in$ $\mathbb{R}^{q \times q}[\zeta, \eta]$ such that

$$
\begin{aligned}
(\zeta & +\eta) Y(\zeta, \eta)+R(\zeta)^{\mathrm{T}} P(\zeta, \eta)+P(\eta, \zeta)^{\mathrm{T}} R(\eta) \\
& =Q(\zeta)^{\mathrm{T}} \Sigma Q(\eta) .
\end{aligned}
$$

A solution to the PLE is then obtained from $P(\zeta, \eta)$ by substituting $\zeta=-\xi$ and $\eta=\xi$, yielding $X(\xi):=P(-\xi, \xi)$. However, this is a rather indirect way of computing a solution $X$ from $Y$. The following proposition shows how an $R$-canonical solution $X$ to the PLE can be expressed directly in terms of a solution $Y$ to the LPLE.

Proposition 4.3. Let $Y \in \mathscr{C}_{R, \mathrm{sym}}^{q \times q}[\zeta, \eta]$ be a solution to the LPLE. Then an R-canonical solution $X \in$ $\mathscr{C}_{R}^{q \times q}[\xi]$ to the PLE is given by

$X(\xi):=-\lim _{|\mu| \rightarrow \infty} \mu R(\mu)^{-\mathrm{T}} Y(\mu, \xi)$.

Moreover, for such $X$ it holds that $(\zeta+\eta) Y(\zeta, \eta)+$ $R(\zeta)^{\mathrm{T}} X(\eta)+X(\zeta)^{\mathrm{T}} R(\eta)=Q(\zeta)^{\mathrm{T}} \Sigma Q(\eta)$.

Propositions 4.1-4.3 show that in order to solve the $P L E$ one may proceed by solving the LPLE first and subsequently constructing an R-canonical solution to the PLE directly from the solution to the LPLE.

If we denote the right-hand side of the LPLE by $\Phi(\zeta, \eta):=Q(\zeta)^{\mathrm{T}} \Sigma Q(\eta)$, then the LPLE can be written compactly as $\mathscr{L}_{R}(Y)=\Phi$, with $\mathscr{L}_{R}$ the Lyapunov operator. From Proposition 3.4, a necessary and sufficient condition for the existence of a unique solution to the LPLE is immediate. It is remarkable that the same condition also characterizes the existence of a unique R-canonical solution to the PLE.

Proposition 4.4. Let $R \in \mathbb{R}^{q \times q}[\xi]$ be nonsingular, let $Q \in \mathbb{R}^{p \times q}[\xi]$ be $R$-canonical and let $\Sigma$ be a $p \times p$ signature matrix defining the PLE and the associated $L P L E$. Let $\lambda_{1}, \ldots, \lambda_{n}$ be the zeros of $\operatorname{det}(R)$. Then the following three statements are equivalent:

1. The following condition is satisfied:

$$
\lambda_{i}+\lambda_{j} \neq 0 \quad \text { for all } i, j=1,2, \ldots, n .
$$

2. The LPLE has a unique (R-canonical) solution.

3. The PLE has a unique R-canonical solution.

For obvious reasons we call condition (4.3) the invertibility condition for the operator $\mathscr{L}_{R}$. Observe that this condition is certainly satisfied when $R$ is Hurwitz (see [14, Theorem 4.8] for an analogous result). The invertibility condition is similar to well-known sufficient conditions for the existence of a solution to the matrix Lyapunov and Sylvester equations (see for example [4, Section VIII.3]).

Before turning to the statement of the iterative procedure for solving the PLE that will be presented in 
the next section, we briefly come back to the issue, raised in the introduction, of a general para-Hermitian right-hand side $Z(\xi)$ for the PLE.

Given $Z(\xi)$, define $\Phi \in \mathbb{R}_{\mathrm{sym}}^{q \times q}[\zeta, \eta]$ as $\Phi(\zeta, \eta):=$ $\left(Z(\zeta)^{\mathrm{T}}+Z(\eta)\right) / 2$; observe that $\Phi(-\xi, \xi)=Z(\xi)$. As in the proof of Proposition 3.2, factor $\Phi$ as $\Phi(\zeta, \eta)=B(\zeta)^{\mathrm{T}} W B(\eta)+R(\zeta)^{\mathrm{T}} G(\zeta, \eta)+G(\eta, \zeta)^{\mathrm{T}} R(\eta)$. Now define $Z_{1}(\xi):=B(-\xi)^{\mathrm{T}} W B(\xi)$ and $Z_{2}(\xi):=$ $R(-\xi)^{\mathrm{T}} G(-\xi, \xi)+G(\xi,-\xi)^{\mathrm{T}} R(\xi)$, whence $Z(\xi)=$ $Z_{1}(\xi)+Z_{2}(\xi)$. Observe that the PLE is linear and consequently its solution space is the direct sum of a particular solution $X_{2}$ to a PLE with right-hand side $Z_{2}$ and the solution space of a PLE with right-hand side $Z_{1}$. Of course $X_{2}$ can be chosen as $G(-\xi, \xi)$. Since $Z_{1}$ can be brought to the form considered in Eq. (1.1) by factoring $W$ as $W=U^{\mathrm{T}} \Sigma U$, issues regarding the solvability of the PLE with general right-hand side $Z$ and the structure of its solution space are still completely addressed by Propositions 4.1 and 4.2.

\section{A recursive procedure for solving the PLE}

In this section we present a recursive procedure for solving the PLE under the assumption that invertibility condition (4.3) is satisfied. The method is conceptually and computationally transparent in the sense that the matrix $R$ need not be transformed to some desired canonical representation, and that the amount of bookkeeping is kept to a minimum. The algorithm is particularly suited for computation in an exact or symbolic context.

The method is inspired by the Faddeev algorithm for computing the resolvent $\left(s I_{n}-A\right)^{-1}$ of an $n \times n$ matrix $A$. (See for example [6, 4, Section IV.4] for a more detailed exposition.) Assume that $A$ is invertible and let $\chi_{A}(\xi)=\operatorname{det}\left(\xi I_{n}-A\right)=\xi^{n}+\chi_{1} \xi^{n-1}+\cdots+$ $\chi_{n-1} \xi+\chi_{n}$ be the characteristic polynomial of $A$. Then $\chi_{n}=(-1)^{n} \operatorname{det}(A) \neq 0$ and also $\chi_{A}(A)=0$ (CayleyHamilton theorem $)$. Note that it follows that $A\left(A^{n-1}+\right.$ $\left.\chi_{1} A^{n-2}+\cdots+\chi_{n-1} I_{n}\right)=-\chi_{n} I_{n}$, whence the inverse of $A$ is given by $A^{-1}=-\left(1 / \chi_{n}\right)\left(A^{n-1}+\chi_{1} A^{n-2}+\cdots+\right.$ $\left.\chi_{n-1} I_{n}\right)$. Observe that the unique solution $\hat{x}=A^{-1} b$ to the linear system of equations $A x=b$ can therefore be computed by the iterative procedure:

$$
\begin{aligned}
& x_{0}:=b, \\
& x_{k}:=A x_{k-1}+\chi_{k} b \quad(k=1,2, \ldots, n-1), \\
& \hat{x}:=-\frac{1}{\chi_{n}} x_{n-1} .
\end{aligned}
$$

In case of the LPLE, we are dealing with a linear system of equations on a finite-dimensional vector space, namely $\mathscr{L}_{R}(Y)=\Phi$. The characteristic polynomial of the Lyapunov operator $\mathscr{L}_{R}$ is described by Eq. (3.2). In order to come up with a procedure for solving the PLE we need only to adapt recursions (5.1)-(5.3) to the case at hand. This yields the main result of this section.

Proposition 5.1. Let $R \in \mathbb{R}^{q \times q}[\xi]$ be nonsingular, let $Q \in \mathbb{R}^{p \times q}[\xi]$ be $R$-canonical and let $\Sigma$ be a $p \times p$ signature matrix. Assume that invertibility condition (4.3) holds. Let $\chi \mathscr{L}_{R}(\xi)=\xi^{d}+\beta_{1} \xi^{d-1}+\cdots+\beta_{d-1} \xi+$ $\beta_{d}$ be the characteristic polynomial of the Lyapunov operator $\mathscr{L}_{R}$ as given by Eq. (3.2), with $d=n(n+1) / 2$ and $n=\operatorname{deg}(\operatorname{det}(R))$. Consider the recursion:

$$
\begin{aligned}
Y_{0}(\zeta, \eta):= & Q(\zeta)^{\mathrm{T}} \Sigma Q(\eta), \\
Y_{k}(\zeta, \eta):= & \mathscr{L}_{R}\left(Y_{k-1}(\zeta, \eta)\right)+\beta_{k} Q(\zeta)^{\mathrm{T}} \Sigma Q(\eta), \\
& \text { for } k=1,2, \ldots, d-1 .
\end{aligned}
$$

Then the two-variable polynomial matrix

$$
Y(\zeta, \eta):=-\frac{1}{\beta_{d}} Y_{d-1}(\zeta, \eta)
$$

yields the unique solution to the LPLE. From $Y$ the unique R-canonical solution $X$ to the PLE is given by

$X(\xi):=-\lim _{|\mu| \rightarrow \infty} \mu R(\mu)^{-\mathrm{T}} Y(\mu, \xi)$.

Knowledge of the characteristic polynomial of $\mathscr{L}_{R}$ is fundamental for application of the algorithm above. Observe that in the context of symbolic or exact computation it is not advisable to compute the characteristic polynomial of $\mathscr{L}_{R}$ from the zeros $\lambda_{i}$ of $\operatorname{det}(R)$ as might suggested by Eq. (3.2). An efficient rational algorithm to compute the coefficients of $\chi \mathscr{L}_{R}$ directly from the coefficients of $\operatorname{det}(R)$ can be designed using Faddeev-type recursions analogous to those of [6, Section 5].

Proposition 5.2. Let $R \in \mathbb{R}^{q \times q}[\xi]$ be nonsingular and let $n=\operatorname{deg}(\operatorname{det}(R))$. Let the $n$ zeros of $\operatorname{det}(R)$ be denoted by $\lambda_{1}, \ldots, \lambda_{n}$ and define $\alpha(\xi)=\xi^{n}+\alpha_{1} \xi^{n-1}+$ $\cdots+\alpha_{n-1} \xi+\alpha_{n}:=\prod_{i=1}^{n}\left(\xi-\lambda_{i}\right)$. In addition, define $\alpha_{k}:=0$ for all $k>n$. Let $d=n(n+1) / 2$ and consider the following three recursions that define the quantities $t_{k}, u_{k}$ and $\beta_{k}$ for $k=1,2, \ldots, d$ :

$$
t_{1}:=-\alpha_{1}, \quad t_{k}:=-\left(k \alpha_{k}+\sum_{\ell=1}^{k-1} t_{\ell} \alpha_{k-\ell}\right),
$$


$u_{1}:=(n+1) t_{1}$,

$u_{k}:=\left(2^{k-1}+n\right) t_{k}+\frac{1}{2} \sum_{\ell=1}^{k-1}\left(\begin{array}{l}k \\ \ell\end{array}\right) t_{\ell} t_{k-\ell}$,

$\beta_{1}:=-u_{1}, \beta_{k}:=-\left(u_{k}+\sum_{\ell=1}^{k-1} u_{\ell} \beta_{k-\ell}\right) / k$.

Then the characteristic polynomial $\chi_{\mathscr{L}_{R}}$ of the Lyapunov operator $\mathscr{L}_{R}$ is given by

$\chi_{\mathscr{L}_{R}}(\xi)=\xi^{d}+\beta_{1} \xi^{d-1}+\cdots+\beta_{d-1} \xi+\beta_{d}$.

Note that the above result shows that the exact computation of the coefficients of the characteristic polynomial of the Lyapunov operator is possible even in cases where the computation of the zeros of $\operatorname{det}(R)$ is infeasible, such as when $R$ depends on symbolic, unspecified parameters.

Remark 1. Algorithm (5.4)-(5.7) involves the computation of the $R$-canonical representative of $(\zeta+\eta) Y_{k-1}(\zeta, \eta)$. It is easy to see that defining $Y_{k}^{\prime}(\xi):=-\lim _{|\mu| \rightarrow \infty} \mu R(\mu)^{-\mathrm{T}} Y_{k}(\mu, \xi)$, it holds that $(\zeta+\eta) Y_{k-1}(\zeta, \eta) \bmod R=(\zeta+\eta) Y_{k-1}(\zeta, \eta)+$ $R(\zeta)^{\mathrm{T}} Y_{k-1}^{\prime}(\eta)+Y_{k-1}^{\prime}(\zeta)^{\mathrm{T}} R(\eta)$. There are good reasons why a crude implementation of this formula for $Y_{k-1}^{\prime}$ should be avoided. First of all, the definition of $Y_{k-1}^{\prime}$ requires the knowledge of the rational matrix $R(\xi)^{-1}$, which in an exact computation context is a delicate issue. Second, a limit operation is required, and this may require a large processing time even for rather small dimensions of the matrices involved. The authors have devised a Faddeev-type recursion that enables the computation of $Y_{k-1}^{\prime}$ with polynomial operations only and which only requires division between the highest-power coefficients of certain univariate polynomials. Such implementation details will be discussed elsewhere; see also [10].

Remark 2. In many cases the matrix $R(\xi)=R_{0}+R_{1} \xi+$ $\cdots+R_{L} \xi^{L}$ has the property that its leading coefficient matrix $R_{L}$ is nonsingular. For example, this always happens for the scalar PLE: $r(-\xi) x(\xi)+x(-\xi) r(\xi)=$ $q(-\xi) q(\xi)$, where $r, q$ and $x \in \mathbb{R}[\xi]$. An algorithm can then be developed that takes advantage of the assumption $\operatorname{det}\left(R_{L}\right) \neq 0$. Full details will be presented elsewhere; see also [10].

\section{Example}

In this section we demonstrate our algorithm by means of a worked example. We consider the solution of a matrix PLE in which the matrices $R, Q$ and $\Sigma$ are given by

$$
\begin{aligned}
& R(\xi)=\left(\begin{array}{cc}
-3+\xi & -6-3 \xi+3 \xi^{2} \\
0 & -2-\xi+\xi^{2}
\end{array}\right), \\
& Q(\xi)=\left(\begin{array}{ll}
0 & 1 \\
1 & 2
\end{array}\right), \quad \Sigma=\left(\begin{array}{ll}
1 & 0 \\
0 & 1
\end{array}\right) .
\end{aligned}
$$

The matrix $R$ is column reduced with column degrees 1 and 2, so that the set of $R$-canonical (one-variable) matrices consists of all matrices with constant entries in the first column and entries of degree at most 1 in the second column (see [7, Theorem 6.3-11]). The matrix $Q$ is obviously $R$-canonical.

The characteristic polynomial of $\mathscr{L}_{R}$ is computed, for instance using the procedure of Proposition 5.2 or by observing that the zeros of $\operatorname{det}(R)$ in this example are equal to $-1,2$ and 3 , as

$$
\begin{aligned}
\chi \mathscr{L}_{R}(\xi)= & \xi^{6}-16 \xi^{5}+85 \xi^{4}-130 \xi^{3}-236 \xi^{2} \\
& +776 \xi-480 .
\end{aligned}
$$

Since the constant term is nonzero, the invertibility condition holds and the PLE is solvable with a unique $R$-canonical solution $X$. Recursion (5.4)-(5.5) generates the following matrices:

$$
\begin{aligned}
& Y_{1}(\zeta, \eta)=\left(\begin{array}{cc}
-10 & -26+2 \eta \\
-26+2 \zeta & -80+5 \eta+5 \zeta
\end{array}\right), \\
& Y_{2}(\zeta, \eta)=\left(\begin{array}{cc}
25 & 96-18 \eta \\
96-18 \eta & 445-75 \eta-75 \zeta+10 \zeta \eta
\end{array}\right),
\end{aligned}
$$$$
Y_{3}(\zeta, \eta)=
$$$$
\left(\begin{array}{cc}
20 & -8+24 \eta \\
-8+24 \zeta & -950+390 \zeta+390 \eta-130 \zeta \eta
\end{array}\right)
$$$$
Y_{4}(\zeta, \eta)=
$$$$
\left(\begin{array}{cc}
-116 & -448+88 \eta \\
-448+88 \zeta & 380-820 \eta-820 \zeta+520 \zeta \eta
\end{array}\right),
$$

$Y_{5}(\zeta, \eta)=$

$$
\left(\begin{array}{cc}
80 & 384-96 \eta \\
384-96 \zeta & 600+600 \eta+600 \zeta-600 \zeta \eta
\end{array}\right) .
$$


It can be verified that

$$
\begin{aligned}
Y(\zeta, \eta) & :=\frac{1}{480} Y_{5}(\zeta, \eta) \\
& =\left(\begin{array}{cc}
\frac{1}{6} & \frac{4}{5}-\frac{1}{5} \eta \\
\frac{4}{5}-\frac{1}{5} \zeta & \frac{5}{4}+\frac{5}{4} \eta+\frac{5}{4} \zeta-\frac{5}{4} \zeta \eta
\end{array}\right)
\end{aligned}
$$

is indeed a solution to the LPLE $\mathscr{L}_{R}(Y(\zeta, \eta))=$ $Q(\zeta)^{\mathrm{T}} \Sigma Q(\eta)$. The unique $R$-canonical solution $X$ to the PLE is computed from $Y$ as described by Eq. (5.7):

$$
X(\xi)=\left(\begin{array}{cc}
-\frac{1}{6} & \frac{-4+\xi}{5} \\
\frac{7}{10} & \frac{23+13 \xi}{20}
\end{array}\right) .
$$

\section{Conclusions}

The algorithm for solving the PLE presented here works directly with the polynomial matrices that constitute the PLE; no preprocessing or transformations to canonical forms are required. The amount of bookkeeping necessary to perform the computations is minimal and the procedure is straightforward to implement. Moreover, the methods employed make the algorithm especially suitable for exact and symbolic computation purposes. Indeed, (5.4)-(5.7) can be applied also when the entries of $R$ or $Q$ depend on unspecified (symbolic) parameters.

The application of the two-variable polynomial framework proposed in this paper to the solution of other polynomial equations relevant for systems and control applications (most urgently the polynomial Sylvester equation) is currently being studied.

\section{Appendix}

Proof of Proposition 2.2. According to [7, Section 6.3] there exists a unimodular matrix $U$ and a column reduced matrix $R^{\prime}$ such that $R^{\prime}=R U$. For $i=1,2, \ldots, q$ let the degree of the $i$ th column of $R^{\prime}$ be denoted by $k_{i}$. Then $\sum_{i=1}^{q} k_{i}=\operatorname{deg}\left(\operatorname{det}\left(R^{\prime}\right)\right)=\operatorname{deg}(\operatorname{det}(R))=n$. Observe that $D^{\prime} \in \mathscr{C}_{R}^{1 \times q}[\xi]$ if and only if $D^{\prime} R^{-1}=$ $D^{\prime} U\left(R^{\prime}\right)^{-1}$ is strictly proper. According to [7, Lemma 6.3-11] this is the case if and only if each of the de- grees of the entries of the $1 \times q$ polynomial row vector $D^{\prime} U$ is strictly less than the degrees of the corresponding columns of $R^{\prime}$, i.e., if and only if for all $i=1,2, \ldots, q$ the $i$ th entry of $D^{\prime} U$ is an element of the $k_{i}$-dimensional space of polynomials in $\xi$ of degree strictly less than $k_{i}$. Thus, $D^{\prime} U$ must be an element of an $n$-dimensional space of $1 \times q$ matrix polynomials; this space is in fact seen to be identical to $\mathscr{C}_{R^{\prime}}^{1 \times q}[\xi]$. Since $U$ is fixed and invertible with a polynomial inverse, the spaces $\mathscr{C}_{R^{\prime}}^{1 \times q}[\xi]$ and $\mathscr{C}_{R}^{1 \times q}[\xi]=\mathscr{C}_{R^{\prime}}^{1 \times q}[\xi] U^{-1}$ have the same dimension $n$.

Proof of Proposition 2.4. First, we assume that $\operatorname{det}(R)$ has $n$ distinct real zeros. This is the case treated in the literature, see [2, Theorem 4-6]. Suppose $\lambda$ is a zero of $\operatorname{det}(R)$ so that $R(\lambda)$ is singular. Then there exists a nonzero vector $v^{\infty}$ in the left kernel of $R(\lambda)$ for which $v^{\infty} R(\lambda)=0$. Consequently, $v^{\infty} R(\xi)=v^{\infty}(R(\xi)-R(\lambda))$. Obviously all entries of $R(\xi)-R(\lambda)$ contain the polynomial factor $\xi-\lambda$, whence $v(\xi):=v^{\infty} R(\xi) /(\xi-\lambda)$ defines a $1 \times q$ matrix polynomial. Since $v(\xi) R(\xi)^{-1}=v^{\infty} /(\xi-\lambda)$ is nonzero and strictly proper we have that $v$ is a nonzero element of $\mathscr{C}_{R}^{1 \times q}[\xi]$. Moreover, we have that $(\xi-\lambda) v(\xi)=$ $v^{\infty} R(\xi)$ and therefore $\xi v(\xi) \bmod R(\xi)=\lambda v(\xi)$. This shows that $\lambda$ is an eigenvalue of the shift operator $S$. Since $\operatorname{deg}(\operatorname{det}(R))=n$ and $\operatorname{dim} \mathscr{C}_{R}^{1 \times q}[\xi]=n$ we have in this case that $\chi_{S}(\xi)=\operatorname{det}(R) / r_{0}$.

In the case where $\operatorname{det}(R)$ has $n$ distinct zeros that possibly are complex, a completely analogous argument can be used if one considers all polynomials involved to be polynomials over $\mathbb{C}$ rather than over $\mathbb{R}$.

Finally, in the general case where $\operatorname{det}(R)$ may have zeros with multiplicity larger than one, a continuity argument can be used to complete the proof. For full details, see [10].

Proof of Proposition 3.2. Let $L$ be the highest power of $\zeta$ or $\eta$ in any entry of $\Phi \in \mathscr{C}_{R \text {, sym }}^{q \times q}[\zeta, \eta]$; then $\Phi$ can be written in a unique way as

$$
\begin{aligned}
\Phi(\zeta, \eta) & =\sum_{i=0}^{L} \sum_{j=0}^{L} \tilde{\Phi}_{i j} \zeta^{i} \eta^{j} \\
& =\left(I_{q}, \zeta I_{q}, \ldots, \zeta^{L} I_{q}\right) \tilde{\Phi}\left(I_{q}, \eta I_{q}, \ldots, \eta^{L} I_{q}\right)^{\mathrm{T}},
\end{aligned}
$$

where $\tilde{\Phi}=\left(\tilde{\Phi}_{i j}\right)$ is an $(L+1) q \times(L+1) q$ block-partitioned symmetric matrix, called the coefficient matrix of $\Phi$. 
Let $\left\{b_{1}, \ldots, b_{n}\right\}$ be a basis for the $n$-dimensional vector space $\mathscr{C}_{R}^{1 \times q}[\xi]$, and define

$B:=\left(\begin{array}{c}b_{1} \\ \vdots \\ b_{n}\end{array}\right)$.

Then the matrix $\left(I_{q}, \xi I_{q}, \ldots, \xi^{L} I_{q}\right)^{\mathrm{T}} \bmod R \in \mathscr{C}_{R}^{1 \times q}[\xi]$ can be written as a product $K B$ for some uniquely determined matrix $K \in \mathbb{R}^{(L+1) q \times n}$. Moreover, there exists $P \in \mathbb{R}^{(L+1) q \times q}[\xi]$ such that $\left(I_{q}, \xi I_{q}, \ldots, \xi^{L} I_{q}\right)^{\mathrm{T}}=$ $K B(\xi)+P(\xi) R(\xi)$.

Consequently, we can write $\Phi(\zeta, \eta)=(K B(\zeta)+$ $P(\zeta) R(\zeta))^{\mathrm{T}} \tilde{\Phi}(K B(\eta)+P(\eta) R(\eta))=B(\zeta)^{\mathrm{T}} W B(\eta)+$ $R(\zeta)^{\mathrm{T}} G(\zeta, \eta)+G(\eta, \zeta)^{\mathrm{T}} R(\eta)$, where $W:=K^{\mathrm{T}} \tilde{\Phi} K \in$ $\mathbb{R}^{n \times n}$ and $G(\zeta, \eta):=P(\zeta)^{\mathrm{T}} \tilde{\Phi}\left(K B(\eta)+\frac{1}{2} P(\eta) R(\eta)\right)$. This shows that $\Phi(\zeta, \eta) \bmod R=B(\zeta)^{\mathrm{T}} W B(\eta)$, because the latter expression is obviously $R$-canonical and $R$-equivalent to $\Phi(\zeta, \eta)$. Conversely, every polynomial matrix of the form $B(\zeta)^{\mathrm{T}} W B(\eta)$ with $W$ an $n \times n$ symmetric matrix, is an element of $\mathscr{C}_{R, \mathrm{sym}}^{q \times q}[\zeta, \eta]$.

Denoting $W=\left(w_{i j}\right)$ with $w_{i j}=w_{j i}$ for $i, j=1,2, \ldots, n$, we have that $B(\zeta)^{\mathrm{T}} W B(\eta)=\sum_{i=1}^{n} w_{i i} b_{i}(\zeta)^{\mathrm{T}} b_{i}(\eta)+$ $\sum_{1 \leqslant i<j \leqslant n} w_{i j}\left[b_{i}(\zeta)^{\mathrm{T}} b_{j}(\eta)+b_{j}(\zeta)^{\mathrm{T}} b_{i}(\eta)\right]$, which expresses $B(\zeta)^{\mathrm{T}} W B(\eta)$ as a linear combination of $n(n+1) / 2$ symmetric two-variable polynomial matrices. To prove that such matrices are linearly independent, consider the expression $B(\zeta)^{\mathrm{T}} W B(\eta)=$ $\sum_{i=1}^{n} \sum_{j=1}^{n} w_{i j} b_{i}(\zeta)^{\mathrm{T}} b_{j}(\eta)=\sum_{j=1}^{n}\left(\sum_{i=1}^{n} w_{i j} b_{i}(\zeta)^{\mathrm{T}}\right)$ $b_{j}(\eta)$. Observe that $B(\zeta)^{\mathrm{T}} W B(\eta)=0$ if and only if $\sum_{i=1}^{n} w_{i j} b_{i}(\zeta)^{\mathrm{T}}=0$ for $j=1,2, \ldots, n$. But this is the case if and only if $w_{i j}=0$ for $i, j=1,2, \ldots, n$. Consequently, we have established a basis for $\mathscr{C}_{R, \mathrm{sym}}^{q \times q}[\zeta, \eta]$ consisting of $n(n+1) / 2$ symmetric two-variable polynomial matrices.

Proof of Proposition 3.4. As in the proof of Proposition 2.4 we first assume that $\operatorname{det}(R)$ has $n$ real distinct zeros, denoted by $\lambda_{1}, \ldots, \lambda_{n}$. To each zero $\lambda_{i}$ of $\operatorname{det}(R)$ there corresponds an eigenvector $v_{i}(\xi)$ of the one-variable shift map $S$ on $\mathscr{C}_{R}^{1 \times q}[\xi]$. Eigenvectors associated with distinct eigenvalues are linearly independent, which provides us with a basis $\left\{v_{1}, \ldots, v_{n}\right\}$ for $\mathscr{C}_{R}^{1 \times q}[\xi]$ consisting of eigenvectors of $S$. According to the proof of Proposition 3.2 a basis for $\mathscr{C}_{R, \mathrm{sym}}^{q \times q}[\zeta, \eta]$ is given by the $n(n+1) / 2$ symmetric two-variable polynomial matrices of the form $v_{i}(\zeta)^{\mathrm{T}} v_{j}(\eta)+v_{j}(\zeta)^{\mathrm{T}} v_{i}(\eta)$, with $1 \leqslant i \leqslant j \leqslant n$. These matrices are also eigenvectors of the operator $\mathscr{L}_{R}$. Indeed, it is easily seen that $(\zeta+\eta)\left(v_{i}(\zeta)^{\mathrm{T}} v_{j}(\eta)+v_{j}(\zeta)^{\mathrm{T}} v_{i}(\eta)\right) \bmod R=\left(\lambda_{i}+\right.$
$\left.\lambda_{j}\right)\left(v_{i}(\zeta)^{\mathrm{T}} v_{j}(\eta)+v_{j}(\zeta)^{\mathrm{T}} v_{i}(\eta)\right)$. This fact implies that the eigenvalues of $\mathscr{L}_{R}$ are $\lambda_{i}+\lambda_{j}, i \leqslant j$, and this proves the claim on the characteristic polynomial of $\mathscr{L}_{R}$.

In the case where $\operatorname{det}(R)$ has $n$ distinct zeros that possibly are complex, a completely analogous argument can be used if one considers all polynomials involved to be polynomials over $\mathbb{C}$ rather than over $\mathbb{R}$. In the general case where $\operatorname{det}(R)$ may have zeros with multiplicity larger than one, a continuity argument can again be used to complete the proof. The details of this can be found in [10].

Proof of Proposition 4.1. Let $X \in \mathbb{R}^{q \times q}[\xi]$ be a solution to the PLE. Define $\Phi(\zeta, \eta):=Q(\zeta)^{\mathrm{T}} \Sigma Q(\eta)-$ $R(\zeta)^{\mathrm{T}} X(\eta)-X(\zeta)^{\mathrm{T}} R(\eta)$. Then obviously $\Phi(\zeta, \eta)$ is symmetric and $\Phi(-\xi, \xi)=0$. According to [14, Theorem 3.1] this implies the existence of a symmetric matrix polynomial $Y \in \mathbb{R}_{\mathrm{sym}}^{q \times q}[\zeta, \eta]$ such that $\Phi(\zeta, \eta)=$ $(\zeta+\eta) Y(\zeta, \eta)$. Observe that $(\zeta+\eta) Y(\zeta, \eta) \bmod R=$ $Q(\zeta)^{\mathrm{T}} \Sigma Q(\eta)$. Observe also that in general $Y$ does not solve the LPLE, since it need not be $R$-canonical. We now show that the $R$-canonical representative $Y^{\prime}$ of $Y$ does solve the LPLE. By definition of $R$-canonicity there exists a matrix $P \in \mathbb{R}^{q \times q}[\zeta, \eta]$ such that $Y(\zeta, \eta)=Y^{\prime}(\zeta, \eta)+R(\zeta)^{\mathrm{T}} P(\zeta, \eta)+P(\eta, \zeta)^{\mathrm{T}} R(\eta)$.

It is then easy to verify, by mere substitution, that $(\zeta+$ $\eta) Y^{\prime}(\zeta, \eta) \bmod R=Q(\zeta)^{\mathrm{T}} \Sigma Q(\eta)$. Hence $Y^{\prime}$ is indeed an $R$-canonical solution to the LPLE.

Conversely, let $Y$ be a solution to the LPLE. Then by definition of $R$-canonicity there exists a matrix $P \in$ $\mathbb{R}^{q \times q}[\zeta, \eta]$ satisfying

$$
\begin{aligned}
(\zeta & +\eta) Y(\zeta, \eta)+R(\zeta)^{\mathrm{T}} P(\zeta, \eta)+P(\eta, \zeta)^{\mathrm{T}} R(\eta) \\
& =Q(\zeta)^{\mathrm{T}} \Sigma Q(\eta) .
\end{aligned}
$$

By substituting $-\xi$ for $\zeta$ and $\xi$ for $\eta$, it follows that $X(\xi):=P(-\xi, \xi)$ yields a solution to the PLE.

Proof of Proposition 4.2. If $X$ is a solution to the PLE and $S$ is skew-para-Hermitian, then it is easy to verify that $X+S R$ is also a solution to the PLE, because the PLE is linear and $R(-\xi)^{\mathrm{T}} S(\xi) R(\xi)+$ $R(-\xi)^{\mathrm{T}} S(-\xi)^{\mathrm{T}} R(\xi)=0$. Therefore, the set $\mathscr{X}_{R} \oplus \mathscr{S} R$ consists entirely of solutions to the PLE.

To see that it contains all solutions, let $X$ be a solution to the PLE and let $X^{\prime}$ be its $R$-canonical representative. Then there exists a polynomial matrix $S \in$ $\mathbb{R}^{q \times q}[\xi]$ such that $X=X^{\prime}+S R$. Since $X$ solves the PLE it holds that

$Q(-\xi)^{\mathrm{T}} \Sigma Q(\xi)$ 


$$
\begin{aligned}
= & R(-\xi)^{\mathrm{T}} X(\xi)+X(-\xi)^{\mathrm{T}} R(\xi) \\
= & R(-\xi)^{\mathrm{T}} X^{\prime}(\xi)+X^{\prime}(-\xi)^{\mathrm{T}} R(\xi) \\
& +R(-\xi)^{\mathrm{T}}\left[S(\xi)+S(-\xi)^{\mathrm{T}}\right] R(\xi) .
\end{aligned}
$$

Upon premultiplication by $R(-\xi)^{-\mathrm{T}}$ and postmultiplication by $R(\xi)^{-1}$ it follows that

$$
\begin{aligned}
R(-\xi)^{-\mathrm{T}} Q(-\xi)^{\mathrm{T}} \Sigma Q(\xi) R(\xi)^{-1} & \\
= & X^{\prime}(\xi) R(\xi)^{-1}+R(-\xi)^{-\mathrm{T}} X^{\prime}(-\xi)^{\mathrm{T}} \\
& +S(\xi)+S(-\xi)^{\mathrm{T}} .
\end{aligned}
$$

Note that the expression on the left-hand side is a strictly proper rational matrix in $\xi$, since $Q$ is $R$-canonical. Also, the first two terms on the right-hand side are strictly proper in $\xi$, since $X^{\prime}$ is $R$-canonical. Hence, $S(\xi)+S(-\xi)^{\mathrm{T}}$ is strictly proper too. Since it is a polynomial matrix, it follows that $S(\xi)+S(-\xi)^{\mathrm{T}}=0$, so $S$ is skew-para-Hermitian. Hence $Q(-\xi)^{\mathrm{T}} \Sigma Q(\xi)=R(-\xi)^{\mathrm{T}} X^{\prime}(\xi)+X^{\prime}(-\xi)^{\mathrm{T}} R(\xi)$ and $X^{\prime}$ solves the PLE. Consequently $X$ is indeed contained in $\mathscr{X}_{R} \oplus \mathscr{S} R$, as was to be proved.

Proof of Proposition 4.3. Let $Y$ be a solution to the LPLE. Proceeding as in the proof of Proposition 3.2, $Y$ can be factored as $Y(\zeta, \eta)=B(\zeta)^{\mathrm{T}} W B(\eta)$ for some $R$-canonical one-variable polynomial $n \times q$ matrix $B$ representing a basis for $C_{R}^{1 \times q}[\xi]$ and some $n \times n$ constant symmetric matrix $W$. Observe that since $B$ is $R$-canonical, $\xi B(\xi) R(\xi)^{-1}$ is proper. Consequently, $B^{\infty}:=\lim _{|\mu| \rightarrow \infty} \mu B(\mu) R(\mu)^{-1}$ is a well-defined constant matrix. It holds that $\xi B(\xi) \bmod R(\xi)=\xi B(\xi)-$ $B^{\infty} R(\xi)$. Consequently,

$$
\begin{aligned}
(\zeta+ & \eta) Y(\zeta, \eta) \\
= & {\left[(\zeta B(\zeta) \bmod R(\zeta))+B^{\infty} R(\zeta)\right]^{\mathrm{T}} W B(\eta) } \\
& +B(\zeta)^{\mathrm{T}} W\left[(\eta B(\eta) \bmod R(\eta))+B^{\infty} R(\eta)\right] \\
= & S(B(\zeta))^{\mathrm{T}} W B(\eta)+B(\zeta)^{\mathrm{T}} W S(B(\eta)) \\
& +R(\zeta)^{\mathrm{T}}\left(B^{\infty}\right)^{\mathrm{T}} W B(\eta) \\
& +B(\zeta)^{\mathrm{T}} W B^{\infty} R(\eta),
\end{aligned}
$$

where $S$ denotes the one-variable polynomial shift operator associated with $R$. Note that the first two terms of the last expression are both $R$-canonical. Consequently, $(\zeta+\eta) Y(\zeta, \eta) \bmod R=$ $S\left(B(\zeta)^{\mathrm{T}}\right) W B(\eta)+B(\zeta)^{\mathrm{T}} W S(B(\eta))=Q(\zeta)^{\mathrm{T}} \Sigma Q(\eta)$. Now observe that the limit defining $X$ in (4.2) exists, since $X(\xi):=-\lim _{|\mu| \rightarrow \infty} \mu R(\mu)^{-\mathrm{T}} Y(\mu, \xi)=$ $-\lim _{|\mu| \rightarrow \infty} \mu R(\mu)^{-\mathrm{T}} B(\mu)^{\mathrm{T}} \Psi B(\xi)=-\left(B^{\infty}\right)^{\mathrm{T}} \Psi B(\xi)$, which is $R$-canonical. Consequently $(\zeta+\eta) Y(\zeta, \eta)+$
$R(\zeta)^{\mathrm{T}} X(\eta)+X(\zeta)^{\mathrm{T}} R(\eta)=Q(\zeta)^{\mathrm{T}} \Sigma Q(\eta)$ and $X$ is an $R$-canonical solution to the PLE.

Proof of Proposition 4.4. Equivalence of (1) and (2) follows from the observation that the LPLE has a unique $R$-canonical solution for every possible choice of the right-hand-side matrix if and only if the operator $\mathscr{L}_{R}$ is invertible. From Proposition 3.4, it follows that this is the case if and only if condition (4.3) holds.

In order to show the contrapositive of implication (3) $\Rightarrow(1)$, we assume that the invertibility condition is not satisfied and henceforth $\mathscr{L}_{R}$ is singular. Consequently there exists a nonzero solution $Y \in$ $\mathbb{R}_{\mathrm{sym}}^{q \times q}[\zeta, \eta]$ to the homogeneous LPLE $\mathscr{L}_{R}(Y)=0$. Applying Proposition 4.3 , we conclude that there exists an $R$-canonical solution $X$ to the homogeneous PLE for which $(\zeta+\eta) Y(\zeta, \eta)+R(\zeta)^{\mathrm{T}} X(\eta)+X(\zeta)^{\mathrm{T}} R(\eta)=0$. This solution $X$ is nonzero, because $(\zeta+\eta) Y(\zeta, \eta)$ obviously is nonzero for nonzero $Y$. It follows that the homogeneous PLE admits nonzero $R$-canonical solutions and so statement (3) does not hold.

Finally, in order to prove implication (2) $\Rightarrow(3)$, we assume that (3) does not hold. If the PLE does not have an $R$-canonical solution, then according to Propositions 4.2 and 4.3 the LPLE also does not have a solution and therefore (2) does not hold. Otherwise, the PLE admits at least two different $R$-canonical solutions, say $X_{1}$ and $X_{2}$. Then $X:=X_{1}-X_{2}$ is a nonzero $R$-canonical solution to the homogeneous PLE. Define $\Phi(\zeta, \eta):=R(\zeta)^{\mathrm{T}} X(\eta)+X(\zeta)^{\mathrm{T}} R(\eta)$. Note that $\Phi$ is nonzero, since $R(\zeta)^{-\mathrm{T}} \Phi(\zeta, \eta) R(\eta)^{-1}=X(\eta) R(\eta)^{-1}+$ $R(\zeta)^{-\mathrm{T}} X(\zeta)^{\mathrm{T}}$ is zero if and only if the one-variable strictly proper rational matrix $X R^{-1}$ is zero, which holds if and only if $X$ is zero. The polynomial matrix $\Phi(\zeta, \eta)$ is symmetric and $\Phi(-\xi, \xi)=0$. Hence, by [14, Theorem 3.1] there exists a nonzero symmetric matrix polynomial $Y \in \mathbb{R}_{\mathrm{sym}}^{q \times q}[\zeta, \eta]$ such that $\Phi(\zeta, \eta)=(\zeta+$ $\eta) Y(\zeta, \eta)$. We conclude that $(\zeta+\eta) Y(\zeta, \eta) \bmod R=0$, directly from the definition of $\Phi$. Hence, the Lyapunov operator admits a nontrivial kernel and (2) does not hold, a contradiction.

Proof of Proposition 5.1. Observe that since $\mathscr{L}_{R}$ maps the finite-dimensional space $C_{R, \mathrm{sym}}^{q \times q}[\zeta, \eta]$ onto itself and since the invertibility condition holds, $\mathscr{L}_{R}$ is bijective. From Proposition 4.1, it follows that solving the PLE is equivalent to finding $Y(\zeta, \eta)$ such that $\mathscr{L}_{R}(Y(\zeta, \eta))=Q(\zeta)^{T} \Sigma Q(\eta)$. Consequently, $Y(\zeta, \eta)=\mathscr{L}_{R}^{-1}\left(Q(\zeta)^{T} \Sigma Q(\eta)\right)$. Recursions (5.4)-(5.6) are the counterpart of the Faddeev sequence-based 
recursion (5.1) - (5.3). The validity of Eq. (5.7) follows immediately from Proposition 4.3.

Proof of Proposition 5.2. For $k=0,1,2, \ldots$, define the quantities $t_{k}$ by $t_{k}=\sum_{i=1}^{n} \lambda_{i}^{k},(k=0,1,2, \ldots)$. Obviously $t_{0}=n$. A relationship between the coefficients $\alpha_{k}$ defined in the proposition and the elementary symmetric functions $t_{k}$ is established by the Newton identities, see [4]: $-k \alpha_{k}=t_{k}+\sum_{\ell=1}^{k-1} t_{\ell} \alpha_{k-\ell},(k=1,2, \ldots, d)$; these are equivalent to recursion (5.8).

Next, consider the polynomial $\chi_{\mathscr{L}_{R}}(\xi)=\xi^{d}+$ $\beta_{1} \xi^{d-1}+\cdots+\beta_{d-1} \xi+\beta_{d}=\prod_{1 \leqslant i \leqslant j \leqslant n}\left(\xi-\left(\lambda_{i}+\lambda_{j}\right)\right)$. Let the quantities $u_{k}$ be defined as the following symmetric functions of the $d$ zeros $\lambda_{i}+\lambda_{j}$ of $\chi_{\mathscr{L}_{R}}$ : $u_{k}=\sum_{1 \leqslant i \leqslant j \leqslant n}\left(\lambda_{i}+\lambda_{j}\right)^{k},(k=0,1,2, \ldots)$. The corresponding Newton identities in this case take the form $-k \beta_{k}=u_{k}+\sum_{\ell=1}^{k-1} u_{\ell} \beta_{k-\ell},(k=1,2, \ldots, d)$. These identities are equivalent to recursion (5.10). Therefore, it only remains to prove that the symmetric functions $t_{k}$ are related to the symmetric functions $u_{k}$ by means of recursion (5.9). This is achieved by considering properties of the Faddeev algorithm of [6, Section 5] for the symmetric matrix Lyapunov equation.

Let the $n \times n$ polynomial matrix $R^{\prime}$ be defined by $R^{\prime}(\xi):=\xi I_{n}-C$, where $C$ is some matrix of which the characteristic polynomial is equal to $\alpha$. Then the operator $\mathscr{L}_{R^{\prime}}$ also has the characteristic polynomial $\chi_{\mathscr{L}_{R}}$. The operator $\mathscr{L}_{R^{\prime}}$ acts on the finite-dimensional space $C_{R^{\prime}, \text { sym }}^{n \times n}[\zeta, \eta]$, which coincides with the space of all $n \times n$ symmetric constant matrices since $R^{\prime}$ is column reduced with all column degrees equal to 1 . It is not difficult to establish that $\mathscr{L}_{R^{\prime}}$ acts on symmetric $n \times n$ constant matrices as

$\mathscr{L}_{R^{\prime}}: Y \mapsto C^{\mathrm{T}} Y+Y C$.

Note that for each $k=0,1, \ldots, d$, the symmetric function $u_{k}$ can be obtained as the trace of the operator $\mathscr{L}_{R^{\prime}}^{k}$, given by

$$
\begin{aligned}
\mathscr{L}_{R^{\prime}}^{k}: Y \mapsto \sum_{\ell=0}^{k}\left(\begin{array}{l}
k \\
\ell
\end{array}\right)\left(C^{\mathrm{T}}\right)^{\ell} Y C^{k-\ell} \\
\quad=\frac{1}{2} \sum_{\ell=0}^{k}\left(\begin{array}{c}
k \\
\ell
\end{array}\right)\left[\left(C^{\mathrm{T}}\right)^{\ell} Y C^{k-\ell}+\left(C^{\mathrm{T}}\right)^{k-\ell} Y C^{\ell}\right] .
\end{aligned}
$$

It follows by the same arguments as used in the proof of [6, Lemma 5.1], that the trace of an operator $M$ of the form $M: Y \mapsto P Y Q^{\mathrm{T}}+Q Y P^{\mathrm{T}}$, acting on $n \times n$ constant symmetric matrices, and with $P$ and $Q$ real matrices of size $n \times n$, is given by $\operatorname{trace}(M)=$ $\operatorname{trace}(P) \operatorname{trace}(Q)+\operatorname{trace}(P Q)$. Hence, the trace $u_{k}$ of $\mathscr{L}_{R^{\prime}}^{k}$ is given by

$$
\begin{aligned}
u_{k} & =\frac{1}{2} \sum_{\ell=0}^{k}\left(\begin{array}{l}
k \\
\ell
\end{array}\right)\left[\operatorname{trace}\left(C^{\ell}\right) \operatorname{trace}\left(C^{k-\ell}\right)+\operatorname{trace}\left(C^{k}\right)\right] \\
& =2^{k-1} t_{k}+\frac{1}{2} \sum_{\ell=0}^{k}\left(\begin{array}{l}
k \\
\ell
\end{array}\right) t_{\ell} t_{k-\ell},
\end{aligned}
$$

for $k=0,1, \ldots, d$. Note that $\operatorname{trace}\left(C^{k}\right)=t_{k}$. Finally, recursion (5.9) follows as $t_{0}=n$.

\section{References}

[1] R.W. Brockett, Finite Dimensional Linear Systems, Wiley, New York, 1970.

[2] P.A. Fuhrmann, Linear Systems and Operators in Hilbert Space, McGraw-Hill, New York, 1981.

[3] P.A. Fuhrmann, A Polynomial Approach to Linear Algebra, Springer, Berlin, 1996.

[4] F.R. Gantmacher, The Theory of Matrices I, Chelsea, New York, 1959.

[5] B. Hanzon, Some new results on and applications of an algorithm of Agashe, in: R.F. Curtain (Ed.), Modelling, Robustness and Sensitivity Reduction in Control Systems, NATO, ASI Series, vol. F 34, Springer, Berlin, 1987.

[6] B. Hanzon, R.L.M. Peeters, A Faddeev sequence method for solving Lyapunov and Sylvester equations, Linear Algebra Appl. 241-243 (1996) 401-430.

[7] T. Kailath, Linear Systems, Prentice-Hall, Englewood Cliffs, NJ, 1980.

[8] R.E. Kalman, Algebraic characterization of polynomials whose zeros lie in certain algebraic domains, Proc. Natl. Acad. Sci. 64 (1969) 818-823.

[9] R.L.M. Peeters, B. Hanzon, Symbolic computation of Fisher information matrices for parametrized state-space systems, Automatica 35 (1999) 1059-1071.

[10] R.L.M. Peeters, P. Rapisarda, A new algorithm to solve the polynomial Lyapunov equation, Report M 99-03, Dept. Math., Universiteit Maastricht, 1999.

[11] A. Talbot, The evaluation of integrals of products of linear system responses - Part I, Quart. J. Mech. Appl. Math. 12 (4) (1959) 488-503.

[12] A. Talbot, The evaluation of integrals of products of linear system responses - Part II. Continued-fraction expansion, Quart. J. Mech. Appl. Math. 12 (4) (1959) 504-520.

[13] H.L. Trentelman, P. Rapisarda, New algorithms for polynomial $J$-spectral factorization, Math. Control, Signals Systems 12 (1999) 24-61.

[14] H.L. Trentelman, J.C. Willems, On quadratic differential forms, SIAM J. Control Opt. 36 (5) (1998) 1703-1749.

[15] J.C. Willems, P.A. Fuhrmann, Stability theory for high order equations, Linear Algebra Appl. 167 (1992) 131-149. 